Canadian Journal of Animal Science Revue canadienne de science animale

Efficacy of Pea Protein Isolate-Alginate Encapsulation on Viability of a Probiotic Bacterium in the Porcine Digestive Tract

\begin{tabular}{|r|l|}
\hline Journal: & Canadian Journal of Animal Science \\
\hline Manuscript ID & CJAS-2016-0090.R1 \\
\hline Manuscript Type: & Article \\
\hline Date Submitted by the Author: & $16-$ Sep-2016 \\
\hline Complete List of Authors: & $\begin{array}{l}\text { Wang, Jing; University of Saskatchewan, Animal and Poultry Science } \\
\text { Nickerson, Micheal; University of Saskatchewan } \\
\text { Low, Nicholas; University of Saskatchewan, Food and Bioproduct Sciences } \\
\text { Van Kessel, Andrew; University of Saskatchewan, Animal \& Poultry Science }\end{array}$ \\
\hline Keywords: & Swine, probiotics, Encapsulation, Microbiota, in vivo \\
\hline \multicolumn{2}{|l}{} \\
\hline
\end{tabular}


Running head: Probiotic encapsulation in swine

\title{
Efficacy of Pea Protein Isolate-Alginate Encapsulation on Viability of a Probiotic Bacterium in the Porcine Digestive Tract
}

\author{
J. Wang, * M.T. Nickerson, $\uparrow$ N.H. Low, $\uparrow$ A.G. Van Kessel ${ }^{* 1}$ \\ *Department of Animal and Poultry Science, University of Saskatchewan, Canada, S7N 5A8 \\ $\dagger$ Department of Food and Bioproduct Sciences, University of Saskatchewan, Canada, S7N 5A8
}

1 Corresponding author: andrew.vankessel@usask.ca 


\begin{abstract}
The aim of this study was to investigate the efficacy of pea protein isolate-alginate capsules (PPCs) on probiotic viability during transit of the porcine gastrointestinal tract. A Lactobacillus reuteri ATCC 53608 isolate selected for rifampin plus streptomycin resistance (LRR) was encapsulated in a pea protein isolate and alginate (LRR-PPC) using an extrusion and crosslinking method prior to freeze drying. An in vitro study in simulated gastric juice showed that encapsulation increased $(P<0.001)$ survival in strong acid. After incorporation into the diet of weaned pigs, LRR were recovered from feces and digesta by selective culture. Fecal shedding of LRR from pigs fed LRR-PPC was higher $(P<0.001)$ than from pigs fed non-encapsulated LRR. Viable LRR counts were not different in homogenized stomach contents, however, higher $(P<0.001)$ counts were observed in distal intestinal contents for pigs fed LRR-PPC. Probiotic encapsulation using pea protein-alginate matrix can protect bacteria during upper intestinal transit improving viability in the distal gut and permitting a broader range of sensitive bacterial species candidates for probiotic application.
\end{abstract}

Keywords: Encapsulation, probiotic, microbiota, swine, in vivo 


\section{INTRODUCTION}

Probiotic bacteria supplementation holds promise to improve animal and human health as an alternative strategy to in-feed/food prophylactic antibiotic use. A prerequisite for such application of probiotics is to maintain the viability of the probiotic organism at a sufficiently high level to be able to colonize the distal gut adequately to affect the host beneficially (Rambaud et al. 1993). Bacteria with probiotic properties may be sensitive to the digestive enzymes, gastric acid, bile salts and other antimicrobial compounds present in the upper gastrointestinal tract (Klemmer et al. 2011; Zhang et al. 2013; Tee et al. 2014) limiting their viability and thus application as oral supplements. Providing additional mechanisms to protect sensitive bacteria against environmental challenges in the gastrointestinal tract could improve the efficacy of existing probiotics and/or permit application of novel probiotic species.

Encapsulation, which is an approach to immobilize and trap probiotic bacteria in a coating matrix, has been shown to improve the viability of probiotic strains during in vitro challenge (Lee and Heo 2000; Klemmer et al. 2011; Sathyabama et al. 2014). Several coating polymer materials have been used for encapsulation such as alginate, chitosan, carrageenan (Annan et al. 2008; Tee et al. 2014). Alginate is an inexpensive, non-toxic, gel-forming material broadly used in encapsulation in the presence of calcium ion (Su et al. 2011; Sathyabama et al. 2014). However, alginate alone did not show sufficient protection of probiotic viability in vitro (Lee and Heo 2000). Milk proteins have been widely used in combination with alginate to improve probiotic protection (Picot and Lacroix 2004; Heidebach et al. 2010). Pea protein isolates are an abundant and low-cost alternative plant protein source which have shown similar protective properties as milk proteins in combination with alginate (Klemmer et al. 2011). 
Although, numerous studies have shown improved bacterial in vitro viability following encapsulation, there has been limited investigations on the effect of encapsulation on probiotic viability in vivo. Rosas-Ledesma (2012) showed that red fluorescence protein-labelled Alteromonadaceae shewanella coated with alginate could be recovered from the ileum of Senegalese sole whereas non-encapsulated A. shewanella could not (Rosas-Ledesma et al. 2012). Similarly, chitosan-coated alginate capsules increased Lactobacillus spp. abundance in mouse (Iyer et al. 2013) and chicken intestine (Rodklongtan et al. 2014). We hypothesized that peaprotein alginate encapsulation of a probiotic would increase the viability of the organism in the digestive tract. Therefore, in the present study, we selected an antibiotic-resistant strain of $L$. reuteri as a model probiotic species, to investigate the efficacy of a pea protein isolate-alginate encapsulation method on probiotic viability in the porcine digestive tract. 


\section{MATERIALS AND METHODS}

All experimental protocols involving animals were approved by the Animal Care Committee of the University of Saskatchewan (\#20110065) and the experiments were performed in accordance with recommendations of the Canadian Council on Animal Care (1993).

\section{Selection and stability of antibiotic-resistant L. reuteri}

To confirm the concentration and class of antibiotic necessary to inhibit growth of resident lactobacilli in pigs, fresh porcine fecal samples $(1 \mathrm{~g})$ from weaned piglets $(n=10)$ were diluted with $9 \mathrm{~g}$ of peptone water [Bacto ${ }^{\mathrm{TM}}$ peptones $1.0 \mathrm{~g} / \mathrm{L}$ (Becton, Dickson and Company, Sparks, MD, USA), $\mathrm{NaCl} 8.5 \mathrm{mM}$ (Fisher Scientific, Ottawa, ON, CA)]. The diluted feces were plated on de Man Rogosa and Sharpe agar (MRS, Becton, Dickson and Company, Sparks, MD, USA) containing rifampin (Sigma-Aldrich, St. Louis, MO, USA) at concentrations of 20, 100 or 200 $\mu \mathrm{g} / \mathrm{mL}$ or containing a combination of rifampin and streptomycin (Sigma-Aldrich, St. Louis, MO, USA) where streptomycin was added at ten times the concentration of rifampin (Pedersen and Tannock 1989; Simpson et al. 2000). Plates were cultured for $48 \mathrm{~h}$ at $37^{\circ} \mathrm{C}$ under anaerobic conditions (GasPak ${ }^{\mathrm{TM}}$ Anaerobic container system, Becton, Dickson and Company, Sparks, MD, USA) for enumeration of live bacteria.

To establish a resistant bacterium, a single colony of L. reuteri ATCC 53608 (purchased from ATCC, USA) was inoculated into $10 \mathrm{~mL}$ MRS broth and cultured for $24 \mathrm{~h}$ at $37^{\circ} \mathrm{C}$ anaerobically. An aliquot of $10 \mu \mathrm{L}$ of starter culture was amplified into $10 \mathrm{~mL}$ fresh MRS broth containing rifampin plus streptomycin at $1 \mu \mathrm{g} / \mathrm{mL}$ broth and $10 \mu \mathrm{g} / \mathrm{mL}$ broth, respectively. This subculture procedure was repeated daily with increased concentration of both antibiotics until a concentration of $400 \mu \mathrm{g} / \mathrm{mL}$ rifampin and $4000 \mu \mathrm{g} / \mathrm{mL}$ streptomycin was achieved. An aliquot 
was then plated on MRS agar containing both antibiotics $(400 \mu \mathrm{g} / \mathrm{mL}$ rifampin and $4000 \mu \mathrm{g} / \mathrm{mL}$ streptomycin) to isolate a single resistant colony for overnight culture and storage in $30 \%$ glycerol $(w / w)$ at $-80^{\circ} \mathrm{C}$.

Single colonies of wild-type $L$. reuteri (LRW) and resistance $L$. reuteri (LRR) were inoculated in $10 \mathrm{~mL}$ MRS broth with antibiotic $(200 \mu \mathrm{g} / \mathrm{mL}$ rifampin $+2000 \mu \mathrm{g} / \mathrm{mL}$ streptomycin, $\mathrm{AB}+$ ) or without antibiotic (AB-) to establish growth kinetics. Optical density (600 $\mathrm{nm})$ and viable counts were measured every 2 and $4 \mathrm{~h}$, respectively in an anaerobic chamber $(80 \%$ $\mathrm{N}_{2}, 10 \% \mathrm{CO}_{2}$, and $10 \% \mathrm{H}_{2}$ ) for $32 \mathrm{~h}$ at $37^{\circ} \mathrm{C}$.

The stability of antibiotic resistance was measured by subculturing of LRR in the absence of antibiotic. The frozen stock was cultured on MRS agar (AB-) and a single colony subsequently inoculated into $10 \mathrm{~mL}$ MRS (AB-) broth followed by anaerobic culture for $24 \mathrm{~h}$ at $37^{\circ} \mathrm{C}$. After 24 h, a $100 \mu \mathrm{L}$ aliquot was plated on MRS (AB+) containing $200 \mu \mathrm{g} / \mathrm{mL}$ rifampin $+2000 \mu \mathrm{g} / \mathrm{mL}$ streptomycin to enumerate viable antibiotic-resistant LRR. A $10 \mu \mathrm{L}$ aliquot of the $24 \mathrm{~h}$ culture was inoculated into fresh $10 \mathrm{~mL}$ MRS (AB-) for another $24 \mathrm{~h}$. This process was repeated every $24 \mathrm{~h}$ for 14 days. Colonies formed on $\mathrm{MRS}(\mathrm{AB}+)$ were enumerated each day to establish retention of antibiotic-resistant LRR.

\section{Preparation of encapsulated and non-encapsulated bacteria}

Early stationary phase (12 to 14 h) LRR cultures were centrifuged, washed with peptone water and either resuspended in 1 volume of $10 \%$ skim milk (LRR-M) or encapsulated in a pea protein isolate (PropulseTM Pea protein isolate, $80 \%$ protein, Nutri-Pea Limited, Portage-laPrairie, MB, Canada)-alginate (Sigma-Aldrich, Oakville, ON, CA) matrix (LRR-PPC). Pea protein-alginate capsules were prepared by an adapted extrusion method as described by 
Klemmer (Klemmer et al. 2011). Briefly, pea protein isolate (PPI) was dissolved into dd $\mathrm{H}_{2} \mathrm{O}(5 \%$ w/w containing 4\% pea protein) at $\mathrm{pH} 8.0$ (adjusted with $1 \mathrm{M} \mathrm{NaOH}$ ) with mechanical stirring for $30 \mathrm{~min}$ in a water bath at $80^{\circ} \mathrm{C}$. The PPI solution was then cooled to room temperature and adjusted to $\mathrm{pH} 7.0$ with $1 \mathrm{M} \mathrm{HCl}$. Alginate was added at $0.6 \%(\mathrm{w} / \mathrm{w})$ and the mixture heated to $80^{\circ} \mathrm{C}$ with mechanical stirring for another 45 min until the alginate was completely dissolved. The pea protein and alginate solution (PAS) was then cooled to room temperature before washed LRR were added at a ratio of 1 part washed bacteria to 20 parts (w/w) PAS with continuous stirring. The PAS was then extruded through a $20 \mathrm{G}$ needle under air pressure and dropped into a cross-link solution $\left[5 \% \mathrm{CaCl}_{2}\right.$ and $1 \%$ Tween 20 (Fisher, New Jersey, USA)]. After a 30 min hardening time, the capsules were filtered by filter paper and collected in aluminum trays. All LRR-PPCs and LRR-M were vacuum freeze-dried for 5 days at $-20^{\circ} \mathrm{C}$ shelf temperature and $50^{\circ} \mathrm{C}$ collector temperature with a pressure of $0.11 \mathrm{mBar}$ (Freezone $6 \mathrm{~L}$ benchtop freeze dry systems, Labconco ${ }^{\circledR}$, USA) and stored at $-80^{\circ} \mathrm{C}$ until use (Figure 1).

\section{In vitro challenge in simulated gastric juice and simulated intestinal juice}

Early stationary phase (12 to $14 \mathrm{~h}$ ) LRW and LRR cultures were harvested and triplicate samples of freshly prepared LRW, LRR and LRR-PPC were suspended to a concentration of approximately $8 \log \mathrm{cfu} / \mathrm{mL}$ in simulated gastric juice [SGJ: $0.08 \mathrm{M} \mathrm{HCl}$ and $0.2 \% \mathrm{NaCl}(\mathrm{w} / \mathrm{v})$, $\mathrm{pH} 1.5$ or $\mathrm{pH} 2.0$ ] at $37^{\circ} \mathrm{C}$ for $2 \mathrm{~h}$ or simulated intestinal juice [SIJ: $1.25 \% \mathrm{NaHCO}_{3}($ Fisher, New Jersey, USA), 0.6\% (w/v) Difco ${ }^{\mathrm{TM}}$ Oxgall (Becton, Dickson and Company, Sparks, MD), 0.09\% Pancreatin (Sigma, Oakville, ON) ] for $3 \mathrm{~h}$ (Klemmer, 2011). After incubation, SGJ treated samples were neutralized to $\mathrm{pH} 7.0$ using $0.1 \mathrm{M} \mathrm{NaOH}$. In order to physically disrupt the capsules and accurately enumerate viable bacteria, samples were homogenized on ice using a 
homogenizer (Fisher Scientific ${ }^{\mathrm{TM}}$ PowerGen ${ }^{\mathrm{TM}}$ Model 125 Homogenizer, USA) at speed 6 $(30,000 \mathrm{rpm})$ for $3 \times 5 \mathrm{~s}$. The samples were then plated on MRS agar and incubated at $37^{\circ} \mathrm{C}$ anaerobically for $48 \mathrm{~h}$ to enumerate the viable L. reuteri.

\section{In vivo Probiotic Delivery in Pigs}

A total of 24 weaned pigs $(8.81 \pm 0.13 \mathrm{~kg}$ body weight, PIC commercial breed $)$ were divided into three treatments ( 4 pigs/pen) and balanced by weight and gender. All the treatment groups received a non-medicated mash feed based on wheat, barley, soybean meal, and corn distillers grains with solubles and meeting the nutrient requirements for the weanling pig (NRC 1998). To control cross contamination, pens assigned to the control treatment group were housed on the opposite end of the room from pens assigned to pigs supplemented with bacteria. Boots were cleared of organic matters and sprayed with ethanol (75\%) after each visit of individual pens. Beginning of $6 \mathrm{~d}$ post-weaning (expermental d 0), pigs were supplemented with LRR-PPC or LRR-M at a level of $10^{6} \mathrm{cfu} / \mathrm{g}$ diet. Pigs in the third treatment group (Control) did not receive probiotic bacterial supplementation. Diets containing probiotic bacteria were prepared fresh each morning for three consecutive days using bacterial aliquots stored at $-80^{\circ} \mathrm{C}$. Water and diets were offered ad libitum throughout the experiment and fed intake recorded daily. Body weight was recorded at the beginning and end of the supplementation period. To confirm probiotic viability, subsamples (5 g) of feed were collected daily at 09:00 from both orts and freshly mixed feed, diluted in $10 \mathrm{~mL}$ peptone water and homogenized on ice. Samples were plated on MRS $\operatorname{agar}(200 \mu \mathrm{g} / \mathrm{mL}$ rifampin $+2000 \mu \mathrm{g} / \mathrm{mL}$ streptomycin $)$ and cultured anaerobically for $48 \mathrm{~h}$ at $37^{\circ} \mathrm{C}$ to permit enumeration of LRR. 


\section{Enumeration of LRR in feces and digesta}

Fecal samples were collected on days $0,1,2$ and 3 from the anus of individual pigs by digital manipulation. Fecal subsamples $(0.2 \mathrm{~g})$ were collected into pre-weighted $15 \mathrm{~mL}$ conical tubes containing $1 \mathrm{~mL}$ peptone water to permit enumeration of viable $L$. reuteri on selective MRS agar $(200 \mu \mathrm{g} / \mathrm{mL}$ rifampin $+2000 \mu \mathrm{g} / \mathrm{mL}$ streptomycin $)$.

All piglets were killed by captive bolt stunning and pithing at experimental day 4. Total contents (without mucosal scrapings) was collected from the stomach, duodenum (the proximal $10 \%$ of small intestinal length), jejunum ( $1 \mathrm{~m}$ before and after intestinal midpoint), ileum (distal $1 \mathrm{~m}$ of the small intestine exclusive of $10 \mathrm{~cm}$ proximal to the ileo-cecal junction), cecum, proximal colon (ileocecal junction to apex of colonic spiral) and distal colon (apex to base of colonic spiral). All contents were collected into separate sterile plastic weight boats, mixed and subsampled. Subsamples $(200 \mathrm{mg})$ from each location except for stomach were collected into pre-weighed $15-\mathrm{mL}$ conical tubes containing $1 \mathrm{~mL}$ peptone water to permit enumeration of viable antibiotic-resistant $L$. reuteri using MRS agar containing antibiotic. In the case of stomach contents, a subsample ( $2 \mathrm{~g})$ was collected into pre-weighed $50-\mathrm{mL}$ conical tubes containing $5 \mathrm{~mL}$ peptone water. Stomach samples were either plated directly or after homogenization on ice (Fisher Scientific ${ }^{\mathrm{TM}}$ PowerGen ${ }^{\mathrm{TM}}$ Model 125 Homogenizer) at speed 6 (30,000 rpm) for 3 x 5s in order to physically disrupt capsules.

\section{Statistical analysis}

The data analysis was processed by SAS 9.1.3 using the mixed procedure (Statistical Analysis Software, version 9.1.3, SAS Inst. Inc, Cary, NC, USA). One-way analysis of variance (ANOVA) was used to assess treatment differences for in vitro experiments. A completely 
randomized design with factorial arrangement was used to assess lactobacillus counts in feed using treatment (LRR-PPC vs. LRR-M) and Time (0 vs. 24 h) as main effects. A repeated measures analysis was used to compare resistant lactobacilli counts in feces and intestinal contents using treatment as a main effect and day post inoculation or gastrointestinal location as repeated sources of variance, respectively. When a significant interaction with the repeated variable was observed, means within the repeated variable were separated by one-way ANOVA. Where treatment effects with more than two levels were significant $(\mathrm{P}<0.05)$, means were separated using Tukey's multiple comparisons. For in vivo experiments, pig was considered the experimental unit.

\section{RESULTS}

\section{Characteristics of antibiotic-resistant L. reuteri}

One strain of $L$. reuteri was isolated that was capable of growth in $400 \mu \mathrm{g} / \mathrm{mL}$ rifampin + $4000 \mu \mathrm{g} / \mathrm{mL}$ streptomycin following culture in the presence of gradually increasing antibiotic concentrations. This level of resistance exceeded the level of resistance observed in swine fecal microbiota. No growth of bacteria was observed in the culture of swine feces on selective MRS agar containing at least $200 \mu \mathrm{g} / \mathrm{mL}$ rifampin $+2000 \mu \mathrm{g} / \mathrm{mL}$ streptomycin (data not shown). The growth of wild-type LRW and LRR in MRS broth with antibiotic $(200 \mu \mathrm{g} / \mathrm{mL}$ rifampin + $2000 \mu \mathrm{g} / \mathrm{mL}$ streptomycin, $\mathrm{AB}+$ ) or without antibiotic (AB-) was followed over $32 \mathrm{~h}$. Stationary phase for all the groups was observed from 10 to $18 \mathrm{~h}$ followed by exponential growth to about $32 \mathrm{~h}$. The growth pattern of wild-type L. reuteri in AB- was similar to resistant L. reuteri in $\mathrm{AB}+$. Wild-type L. reuteri did not grow in AB+. After daily subculture of LRR for 14 days in AB- 
MRS broth, the number of viable LRR recovered on the AB+ MRS agar remained consistent for each day at $9 \log \mathrm{cfu} / \mathrm{mL}$ broth indicating no loss of resistance.

\section{In vitro challenge in simulated gastric juice and simulated intestinal juice}

After $2 \mathrm{~h}$ incubation in SGJ ( $\mathrm{pH} 2.0)$ and $3 \mathrm{~h}$ in SIJ $(\mathrm{pH}=7.3)$, there was no difference in the reduction of viability of LRW, LRR or LRR-PPC (Table 1). However, after samples were challenged in SGJ ( $\mathrm{pH}$ 1.5) for $2 \mathrm{~h}$, a significant reduction in viability occurred for nonencapsulated LRW and LRR (3.54 \pm 0.06 and 3.51 $\pm 0.09 \log \mathrm{cfu} / \mathrm{g}$, respectively) compared to LRR-PPC group $(1.13 \pm 0.06 \log \mathrm{cfu} / \mathrm{g})$.

\section{Probiotic viability in feed}

Viable count of L. reuteri in freshly prepared feed and in feed recovered from feeding troughs after $24 \mathrm{~h}$ on each of the three experimental supplementation days is shown in Table 2. The viable count was not different for feed supplemented with LRR-M or LRR-PPC. However, there was a significant reduction $(P<0.001)$ of viable bacteria after $24 \mathrm{~h}$ for both LRR-PPC and LRR-M group. No antibiotic-resistant bacteria were recovered from the control diet.

\section{Probiotic shedding in feces}

Feed intake per pig was $245 \pm 14 \mathrm{~g} / \mathrm{d}$, and not different among the three treatment groups for each day of supplementation. The number of antibiotic-resistant lactobacilli recovered in feces on each day of the study is shown in Table 3. There was no recovery of antibiotic-resistant lactobacilli on day 0 for all groups. Statistical comparison of antibiotic-resistant lactobacilli counts in feces from days 1 to 3 in LRR-M and LRR-PPC groups (excluding Control group) 
using a repeated measures approach, indicated a significant $(P<0.01)$ effect of treatment and experimental day and a trend $(P<0.1)$ towards a treatment by day interaction. Fecal resistant lactobacilli count from the LRR-M group was significantly $(P<0.001)$ lower compared with the count in feces from LRR-PPC pigs during each of the $3 \mathrm{ds}$ in the feeding period. The shedding of antibiotic resistant lactobacilli increased $(P<0.05)$ on day 2 and day 3 compared with day 1 independent of how LRR were administered (LRR-M or LRR-PPC). A trend towards a significant treatment by day interaction suggested that the increase in fecal shedding of resistant lactobacilli was greater in LRR-PPC as compared to LRR-M. No fecal shedding of resistant lactobacilli was observed on experimental day 1 in the Control group. However, two Control pigs shed antibiotic-resistant lactobacilli at $4.60 \mathrm{log} \mathrm{cfu} / \mathrm{g}$ on day 2 and two pigs shed resistant lactobacilli at 4.62 and $4.60 \mathrm{log} \mathrm{cfu} / \mathrm{g}$ on day 3 . Only one pig shed resistant lactobacilli on both days.

\section{Enumeration of viable LRR in digesta}

Figure 2 shows counts of viable antibiotic-resistant lactobacilli in intestinal contents recovered along the gastrointestinal tract. As expected, counts in control pigs were below $2 \log$ $\mathrm{cfu} / \mathrm{g}$ in all locations. Interestingly, in contents from stomach and duodenum, viable antibioticresistant lactobacilli counts were higher $(P<0.001)$ in LRR-M compared to LRR-PCC pigs. In contrast, antibiotic-resistant lactobacilli counts in the distal small intestine, cecum and colon were higher $(P<0.001)$ in LRR-PPC compared to LRR-M pigs. Homogenization of the contents from stomach increased lactobacilli counts in stomach contents from LRR-PPC pigs without affecting the counts for LRR-M pigs such that homogenized counts were not different between the two treatment groups. 


\section{DISCUSSION}

Encapsulation has been shown to provide protection to a variety of probiotic bacteria during in vitro challenge with simulated gastric juice and bile salts compared to non-encapsulated bacteria (Guérin et al. 2003; Piatek et al. 2012; Lotfipour et al. 2012; Zhang et al. 2013; Cook et al. 2014). Work has also shown improved bacteria viability in acid food products following encapsulation (Martoni et al. 2007; Ortakci and Sert 2012). However, there are very few studies establishing the efficacy of encapsulation technologies on the viability of probiotic bacteria in the intestinal tract. One limitation of investigating the viability of encapsulated probiotics is the difficulty in the selective enumeration of the encapsulated probiotic strain. Traditional selective culture methods are generally unable to differentiate probiotic bacterial strains from bacteria commonly found in the digestive tract. Furthermore, while molecular methods such as qPCR demonstrate improved selectivity, differentiation of live and dead bacteria remains difficult with this approach. In the current study, L. reuteri was selected as a model probiotic bacterium which has been reported as possessing probiotic properties including secretion of the antimicrobial protein reuterin (Muthukumarasamy et al. 2006) and improving animal growth performance (Agustina et al. 2013). By using natural selection, a strain of L. reuteri with stable resistance to antibiotics at concentrations exceeding resistance in lactobacilli colonizing the pig digestive tract was identified. This strain of L. reuteri allowed specific enumeration of viable probiotic in porcine feces and gastrointestinal tract after supplementation in feed following encapsulation in a pea protein isolate-alginate matrix or not.

\section{In vitro challenge}

Generally, lactic acid bacteria show good acid tolerance (van de Guchte et al. 2002). In this study, both wild-type L. reuteri ATCC 53608 and the antibiotic-resistant strain, were tolerant to 
challenge with simulated gastric juice at $\mathrm{pH}$ 2.0. However, both strains became susceptible when challenged under SGJ at $\mathrm{pH}$ 1.5. The significant protection observed when the $\mathrm{pH}$ of SGJ was lowered to 1.5 is consistent with the other studies investigating encapsulation of Lactobacillus spp. (Muthukumarasamy et al. 2006; Hassan and Rasco 2014). Further, PPCs have provided acid protection in other probiotic strains. Klemmer (2011) demonstrated the PPC significantly improved acid tolerance of Bifidobacterium adolescentis that was otherwise highly susceptible to challenge in SGJ (pH 2.0) (Klemmer et al. 2011). In the case of simulated intestinal juice (SIJ) challenge, bile salts and pancreatin did not affect the viability of L. reuteri used in this study. Instead, SIJ lead to enlarged capsules and may have facilitated the release of encapsulated bacteria (Klemmer 2011). Given the resistance to SIJ observed for the non-encapsulated $L$. reuteri strain used in this study, it is not surprising that no further enhancement in resistance was observed after encapsulation.

\section{In vivo probiotic delivery using pea protein isolate-alginate capsules}

In order to confirm that pigs received the same dose of viable probiotic, viability was determined both immediately after mixing with feed and after $24 \mathrm{~h}$ in the feed troughs. L. reuteri demonstrated significant loss of viability of approximately $1 \log \mathrm{cfu} / \mathrm{g}$ feed during $24 \mathrm{~h}$ in feed at room temperature independent of whether the probiotic was encapsulated or not. Thus, although this observation confirmed that pigs received the same dose of viable probiotic, lack of protection from loss of viability during storage in feed is a significant obstacle to the commercial application of some probiotic strains including lactobacilli, which was not improved by pea protein-alginate encapsulation (Weinbreck et al. 2010). Encapsulation has been previously shown to improve the viable shelf life of probiotic bacteria when stored frozen but not on storage 
at room temperature (Heidebach et al. 2010). Given the typical storage times for prepared feeds, loss of $1 \log$ cfu per gram feed per day would not be commercially acceptable, limiting application to mixing with probiotic immediately prior to feeding.

Fecal shedding of antibiotic-resistant L. reuteri was observed within $24 \mathrm{~h}$ of supplementation consistent with expected. Transit time in the pig digestive tract could be vary by the diet composition and individual pigs and could take from 20 to $102 \mathrm{~h}$ to reach to the rectum in pig (Latymer et al. 1990; Kim et al. 2007). Encapsulation appeared to increase the number of viable L. reuteri shed in feces within $24 \mathrm{~h}$ compared to non-encapsulated and supported a further increase in shedding of approximately $0.5 \log \mathrm{cfu} / \mathrm{g}$ feces over the $3 \mathrm{~d}$ feeding period. The observation suggested that encapsulation improved viable probiotic delivery to the distal gastrointestinal tract as previously observed in mice (Iyer et al. 2013). It is unclear why shedding may have increased over time to a greater extent in the encapsulated group. It is unlikely that the efficacy of the capsules improved but perhaps, the delivery of viable bacteria in higher number affected the dynamics of microbial colonization in this complex community permitting L. reuteri to occupy a larger niche. Finally, although efforts were made to minimize cross contamination of the probiotic between control and supplemented pigs, low counts of viable antibiotic-resistant lactobacilli were observed in feces of some control pigs $48 \mathrm{~h}$ after initiation of the experiment. Similar low levels were found in digesta collected after $3 \mathrm{~d}$ of feeding. Those contaminations in the control group may result from transport on air particles within the room or inadvertent contamination carried between pens by staff. Clearly, the design of studies comparing probiotic supplemented groups and control groups should be carefully considered to minimize transfer of the viable organism to control pigs. 
Enumeration of antibiotic-resistant L. reuteri in digesta collected along the length of the pig gastrointestinal tract indicated an interesting pattern. Whereas as viable antibiotic-resistant lactobacilli counts were relatively static in all locations in LRR-M pigs, counts in LRR-PPC pigs were below LRR-M in proximal regions and above LRR-M in distal regions. Although increased probiotic counts in distal locations for pigs fed LRR-PPC was consistent with higher levels of fecal shedding, low counts in proximal regions were unexpected. However, visual observation indicated the presence of intact capsules in the stomach that were present in declining abundance from proximal to distal regions of the small intestine. The few capsules observed in the ileum appeared swollen and soft relative to the stomach. Although there is limited research on the in vivo delivery of encapsulated probiotic, in vitro results have shown the release of Bifidobacterium adolescentis from pea protein-alginate capsules, formulated similarly to the current study, slowly in simulated intestinal juice (SIJ) over a 3-h period (Klemmer et al. 2011). The release mechanism of PPC was proposed to be mediated by the osmotic change, cleavage of amide bonds by pepsin and trypsin, and physical pressure. When stomach chyme from LRR-PPC pigs was homogenized to disrupt the capsules, similar bacteria counts were observed compared to the LRR-M group without $\mathrm{pH}$ adjusted to neutral for the stomach fluids. This increase in counts is consistent with our in vitro experience regarding enumeration of encapsulated bacteria where homogenization of capsules increased the recovery of viable counts enumerated on agar by 1 to $2 \log$ cfu/g likely via physical disruption permitting bacteria dispersion on the plate. Given the observation of capsules along the length of the small intestine, it is likely that antibiotic resistant L. reuteri enumerated in these locations without homogenization underestimated total counts in LRR-PPC pigs. 
Although disruption of capsules increased counts in the stomach to the same level of nonencapsulated bacteria, no increase in viability of antibiotic-resistant lactobacilli was evident in either stomach or upper small intestine. Because the $\mathrm{pH}$ of stomach contents from this study was above $\mathrm{pH} 3.0$ at the time of euthanasia (data not shown), these observations are consistent with the tolerance of our L. reuteri strain to SGJ ( $\mathrm{pH} 2.0$ ). However, the results do not establish a mechanism by which the capsules improved L. reuteri colonization in distal gut locations. Interestingly, acid tolerance in lactic acid bacteria is related to $\mathrm{H}+-\mathrm{ATPase}$ activity $(\mathrm{H}+$ pump) (Matsumoto et al. 2004) while the survival mechanism from bile is unclear but may be related to triggering removal by an efflux pump (Gunn 2000). Thus, both acid and bile tolerance mechanisms require metabolic adaptations and ATP consumption. It is possible that while capsules may not improve viability, they could minimize the energy expenditure and metabolic adaptations required to survive in the upper gastrointestinal tract environment, improving their ability to compete for space and nutrients in the distal gut. Partial support for this could be extrapolated from the observation that loss of viable $L$. reuteri occurred when the $\mathrm{pH}$ of SGJ was lowered to 1.5 .

\section{CONCLUSION}

An antibiotic-resistant L. reuteri was selected to establish the efficacy of pea protein alginate capsules for in vivo delivery of a probiotic to the gastrointestinal tract of pigs. Although L. reuteri are resistant to acid conditions and bile found in the stomach and duodenum, respectively, encapsulation improved probiotic counts in the distal gastrointestinal tract and shed in feces. Encapsulation may be a viable approach to expanding the taxonomic repertoire of bacteria suitable for commercial probiotic application to include strains sensitive to 
environmental conditions in the upper gastrointestinal tract. Loss of probiotic viability in the feed bunk, however, was not improved by encapsulation. Whether encapsulation could also improve probiotic shelf life under controlled conditions and or improve resistance to feed processing will require further investigation.

Acknowledgment: This study was supported by Agriculture Development Fund. Jing Wang was supported by China Scholarship Council during her Ph.D. study. The assistant of Jason Marshall, Dr. Wolfgang Koester, Dr. Daniel Petri, Laurie Thomson and Prairie Swine Center staff are gratefully acknowledged.

Conflict of Interest: Authors declare no conflict of interest. 


\section{REFERENCES}

Agustina, R., Bovee-Oudenhoven, I.M., Lukito, W., Fahmida, U., van de Rest, O., Zimmermann, M.B., Firmansyah, A., Wulanti, R., Albers, R., van den Heuvel, E.G. and Kok, F.J. (2013) Probiotics Lactobacillus reuteri DSM 17938 and Lactobacillus casei CRL 431 modestly increase growth, but not iron and zinc status, among Indonesian children aged 1-6 years. J. Nutr. 143, 1184-1193.

Annan, N.T., Borza, A.D. and Hansen, L.T. (2008) Encapsulation in alginate-coated gelatin microspheres improves survival of the probiotic Bifidobacterium adolescentis $15703 \mathrm{~T}$ during exposure to simulated gastro-intestinal conditions. Food Res. Int. 41, 184-193.

Cook, M.T., Tzortzis, G., Charalampopoulos, D. and Khutoryanskiy, V.V. (2014) Microencapsulation of a synbiotic into PLGA/alginate multiparticulate gels. Int. J. Pharm. 466, 400-408.

Guérin, D., Vuillemard, J.-C. and Subirade, M. (2003) Protection of bifidobacteria encapsulated in polysaccharide-protein gel beads against gastric juice and bile. J. Food Prot. 66, 2076-2084.

Gunn, J.S. (2000) Mechanisms of bacterial resistance and response to bile. Microbes Infect. 2, 907-913. 
Hassan, A. and Rasco, B. (2014) Microencapsulation, survival and adherence studies of indigenous probiotics. Afr. J. Microbiol. Res. 8, 766-775.

Heidebach, T., Först, P. and Kulozik, U. (2010) Influence of casein-based microencapsulation on freeze-drying and storage of probiotic cells. J. Food Eng. 98, 309-316.

Iyer, C., Phillips, M. and Kailasapathy, K. (2013) Survival and release of probiotic bacteria from chitosan-coated alginate-starch capsules in mice gastro-intestinal tract and effect on faecal flora and immune parameters in mice. Int. J. Ferm. Foods. 2, 19-31.

Kim, B.G., Lindemann, M.D., Cromwell, G.L., Balfagon, A. and Agudelo, J.H. (2007) The correlation between passage rate of digesta and dry matter digestibility in various stages of swine. Livest Sci. 109, 81-84.

Klemmer, K.J. (2011) Synbiot encapsulation employing a pea protein-alginate matrix: University of Saskatchewan Saskatoon.

Klemmer, K.J., Korber, D.R., Low, N.H. and Nickerson, M.T. (2011) Pea protein-based capsules for probiotic and prebiotic delivery. Int. J. Food Sci. Tech. 46, 2248-2256.

Latymer, E.A., Low, A., Fadden, K., Sambrook, I., Woodley, S.C. and Keal, H. (1990) Measurement of transit time of digesta through sections of gastrointestinal tract of pigs fed with 
diets containing various sources of dietary fibre (non-starch polysaccharides). Arch. Anim. Nutr. 40, 667-680.

Lee, K.-Y. and Heo, T.-R. (2000) Survival of Bifidobacterium longum immobilized in calcium alginate beads in simulated gastric juices and bile salt solution. Appl. Environ. Microbl. 66, 869873.

Lotfipour, F., Mirzaeei, S. and Maghsoodi, M. (2012) Preparation and characterization of alginate and psyllium beads containing Lactobacillus acidophilus. Sci. World J. 2012,1-8

Martoni, C., Bhathena, J., Jones, M.L., Urbanska, A.M., Chen, H. and Prakash, S. (2007) Investigation of microencapsulated BSH active Lactobacillus in the simulated human GI tract. J. Biomed. Biotechnol. 2007.13684

Matsumoto, M., Ohishi, H. and Benno, Y. (2004) H+-ATPase activity in Bifidobacterium with special reference to acid tolerance. Int. J. Food Microbol. 93, 109-113.

Muthukumarasamy, P., Allan-Wojtas, P. and Holley, R.A. (2006) Stability of Lactobacillus reuteri in different types of microcapsules. J. Food Sci. 71, M20-M24.

NRC. 1998. Nutrient Requirements of Swine. 10th rev. ed. Natl. Acad. Press, Washington, DC. 
Ortakci, F. and Sert, S. (2012) Stability of free and encapsulated Lactobacillus acidophilus ATCC 4356 in yogurt and in an artificial human gastric digestion system. J. Dairy Sci. 95, 69186925.

Pedersen, K. and Tannock, G. (1989) Colonization of the porcine gastrointestinal tract by lactobacilli. Appl. Environ. Microb. 55, 279-283.

Piatek, J., Gibas-Dorna, M., Olejnik, A., Krauss, H., Wierzbicki, K., Zukiewicz-Sobczak, W. and Glowacki, M. (2012) The viability and intestinal epithelial cell adhesion of probiotic strain combination in vitro study. Ann. Agric. Environ. Med. 19, 99-102

Picot, A. and Lacroix, C. (2004) Encapsulation of bifidobacteria in whey protein-based microcapsules and survival in simulated gastrointestinal conditions and in yoghurt. Int. Dairy J. 14, 505-515.

Rambaud, J.C., Bouhnik, Y., Marteau, P. and Pochart, P. (1993) Manipulation of the human gut microflora. Proc. Nutr. Soc. 52, 357-366.

Rodklongtan, A., La-ongkham, O., Nitisinprasert, S. and Chitprasert, P. (2014) Enhancement of Lactobacillus reuteri KUB-AC5 survival in broiler gastrointestinal tract by microencapsulation with alginate-chitosan semi-interpenetrating polymer networks. J. Appl. Microbiol. 117, 227-238. 
Rosas-Ledesma, P., León-Rubio, J.M., Alarcón, F.J., Moriñigo, M.A. and Balebona, M.C. (2012)

Calcium alginate capsules for oral administration of fish probiotic bacteria: assessment of optimal conditions for encapsulation. Aquacult. Res. 43, 106-116.

Sathyabama, S., Kumar, M.R., Devi, P.B., Vijayabharathi, R. and Priyadharisini, V.B. (2014) Co-encapsulation of probiotics with prebiotics on alginate matrix and its effect on viability in simulated gastric environment. Lwt Food Sci. Technol. 57, 419-425.

Simpson, J.M., McCracken, V.J., Gaskins, H.R. and Mackie, R.I. (2000) Denaturing gradient gel electrophoresis analysis of $16 \mathrm{~S}$ ribosomal DNA amplicons to monitor changes in fecal bacterial populations of weaning pigs after introduction of Lactobacillus reuteri strain MM53. Appl. Environ. Microb. 66, 4705-4714.

Su, R., Zhu, X.L., Fan, D.D., Mi, Y., Yang, C.Y. and Jia, X. (2011) Encapsulation of probiotic Bifidobacterium longum BIOMA 5920 with alginate-human-like collagen and evaluation of survival in simulated gastrointestinal conditions. Int. J. Biol. Macromol. 49, 979-984.

Tee, W., Nazaruddin, R., Tan, Y. and Ayob, M. (2014) Effects of encapsulation on the viability of potential probiotic Lactobacillus plantarum exposed to high acidity condition and presence of bile salts. Food Sci. Technol. Int. 20, 399-404.

Van de Guchte, M., Serror, P., Chervaux, C., Smokvina, T., Ehrlich, S.D. and Maguin, E. (2002) Stress responses in lactic acid bacteria. A. Van Leeuw. 82, 187-216. 
Weinbreck, F., Bodnár, I. and Marco, M. (2010) Can encapsulation lengthen the shelf-life of probiotic bacteria in dry products? Int. J. Food Microbiol. 136, 364-367.

Zhang, F., Li, X.Y., Park, H.J. and Zhao, M. (2013) Effect of microencapsulation methods on the survival of freeze-dried Bifidobacterium bifidum. J. Microencapsul. 30, 511-518. 


\section{TABLES AND FIGURES}

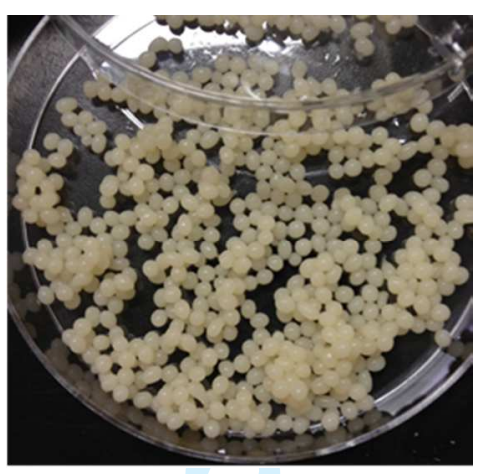

A

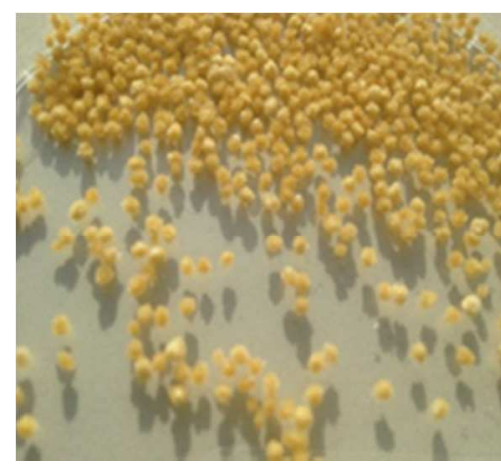

B

Figure 1. Pea protein isolate-alginate capsules (A: Wet capsules after cross-linking and filtering B: Freeze dried capsules). 
Table 1. Reduction in viable counts $(\log \mathrm{cfu} / \mathrm{g})$ of freshly prepared wild type (LRW), antibiotic-resistant (LRR) and encapsulated resistant L. reuteri (LRR-PPC) before or after challenge in simulated gastric juice (SGJ, $\mathrm{pH} 2.0$ and $\mathrm{pH} 1.5$ ) for $2 \mathrm{~h}$ and simulated intestinal juice (SIJ, $\mathrm{pH}=7.3$ ) for $3 \mathrm{~h}^{\mathrm{a}}$.

\begin{tabular}{llllll}
\hline & $\mathrm{pH}$ & LRR-PPC & \multicolumn{1}{c}{ LRR } & \multicolumn{1}{c}{ LRW } & $P$ \\
\hline SGJ & 2 & $1.02 \pm 0.01$ & $1.04 \pm 0.23$ & $1.06 \pm 0.08$ & 0.9721 \\
& 1.5 & $1.13 \pm 0.06 a$ & $3.51 \pm 0.09 b$ & $3.54 \pm 0.06 c$ & $<0.001$ \\
SIJ & 7.3 & $0.04 \pm 0.09$ & $0.06 \pm 0.45$ & $0.10 \pm 0.23$ & 0.9805 \\
\hline
\end{tabular}

Note: Means with a different letter in the same row were significantly different at $P<0.001$ level.

${ }^{\mathrm{a}}$ Results are Mean $\pm \mathrm{SEM}$ for triplicate determinations. 
Table 2. Number (log cfu/g feed) of antibiotic-resistant bacteria enumerated on $\mathrm{AB}+\mathrm{MRS}^{\mathrm{a}}$ agar for freshly prepared feed and feed recovered after $24 \mathrm{~h}$ for freeze dried antibiotic-resistant L. reuteri with milk (LRR-M) and antibiotic-resistant L. reuteri coated with pea protein isolate-alginate capsules (LRR-PPC) supplemented diets for consecutively 3 days $^{\mathrm{b}}$.

\begin{tabular}{lccccccc}
\hline & \multicolumn{2}{c}{ Treatment } & \multicolumn{2}{c}{ Time (h) } & \multicolumn{2}{c}{$P$} \\
\cline { 2 - 8 } Enumeration & LRR-PPC & LRR-M & 0 & 24 & Treatment & Time & Interaction \\
\cline { 2 - 8 } & $5.88 \pm 0.2$ & $5.89 \pm 0.15$ & $6.29 \pm 0.08$ & $5.48 \pm 0.06$ & 0.9428 & $<0.001$ & 0.3414 \\
\hline
\end{tabular}

${ }^{\mathrm{a}} \mathrm{AB}+\mathrm{MRS}$ : de Man, Rogosa and Sharpe media containing rifampin $(200 \mu \mathrm{g} / \mathrm{mL})$ and streptomycin $(2000$ $\mu \mathrm{g} / \mathrm{mL})$.

${ }^{b}$ Results are Mean \pm SEM for triplicate determinations. 
Table 3. Antibiotic-resistant lactobacilli (log cfu/g feces) in fecal samples from pigs fed diets supplemented with freeze dried antibiotic-resistant L. reuteri with milk (LRR-M) or antibioticresistant $L$. reuteri coated with pea protein isolate-alginate capsules (LRR-PPC) ${ }^{\mathrm{a}}$.

\begin{tabular}{|c|c|c|c|c|}
\hline \multirow{2}{*}{$\begin{array}{l}\text { Day post } \\
\text { inoculation }\end{array}$} & \multicolumn{3}{|c|}{ Treatment } & \multirow[b]{2}{*}{ Control $^{b}$} \\
\hline & LRR-M & LRR-PPC & Overall & \\
\hline Day 0 & n.d. ${ }^{\mathrm{c}}$ & n.d. & n.d. & n.d. \\
\hline Day 1 & $4.96 \pm 0.13$ & $5.42 \pm 0.02$ & $5.19 \pm 0.35 a$ & n.d. \\
\hline Day 2 & $5.29 \pm 0.22$ & $6.02 \pm 0.11$ & $5.65 \pm 0.61 b$ & $1.00 \pm 0.61$ \\
\hline Day 3 & $5.13 \pm 0.16$ & $6.32 \pm 0.19$ & $5.72 \pm 0.77 b$ & $0.53 \pm 0.50$ \\
\hline Overall & $5.13 \pm 0.10 a$ & $5.92 \pm 0.11 b$ & & $0.51 \pm 0.28$ \\
\hline \multicolumn{5}{|c|}{$\begin{array}{l}\text { Note: Means with a different letter in the same row were significantly different at } P<0.001 \\
\text { level. }\end{array}$} \\
\hline \multicolumn{5}{|c|}{$\begin{array}{l}{ }^{a} \text { Results are Mean } \pm \text { SEM }(n=8) \text {. Data were analyzed using Proc Mixed procedure using } \\
\text { treatment as a main effect and day post inoculation as a repeated source of variation. Treatment } \\
P<0.001 \text {, Day } P=0.0035 \text {, Treatment X Day } P=0.0857 \text {. }\end{array}$} \\
\hline \multicolumn{5}{|c|}{$\begin{array}{l}\mathrm{b} \text { Data from the Control group was not included in the statistical analysis. On Day } 2 \text {, two pigs } \\
\text { out of eight both shed antibiotic resistance lactobacilli at } 4.60 \log \mathrm{cfu} / \mathrm{g} \text {. On day } 3 \text {, two pigs out } \\
\text { of eight pigs shed antibiotic resistance lactobacilli with a mean of } 4.60 \text { and } 4.62 \mathrm{log} \mathrm{cfu} / \mathrm{g} \text {, } \\
\text { respectively. Only one pig shed resistant lactobacilli on both days. }\end{array}$} \\
\hline
\end{tabular}




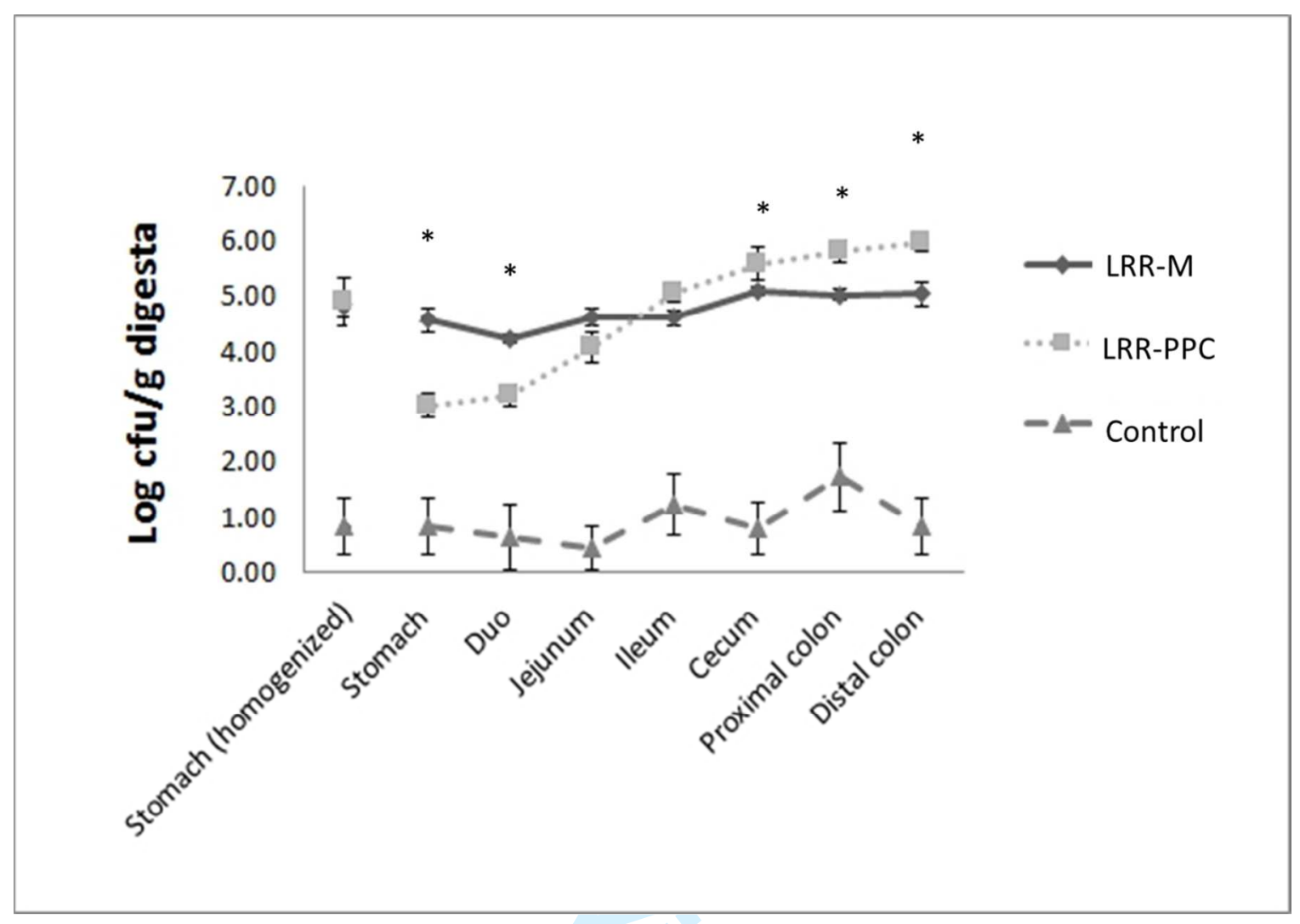

Figure 2. Antibiotic-resistant lactobacilli (log cfu/g digesta) enumerated in digesta ( $\mathrm{n}=8)$ from pigs fed the control diet or diets supplemented with freeze dried antibiotic-resistant L. reuteri with milk (LRR-M) and antibiotic-resistant L. reuteri coated with pea protein isolate-alginate capsules (LRR-PPC). Comparison of LRR-M and LRR-PPC treatment groups by repeated measures indicated Treatment by Location Interaction $P<0.01$. (*within location $P<0.05$ between LRR-M and LRR-PPC). 